\title{
Hematoma duodenal intramural post biopsia endoscópica: una complicación inusual. Reporte de un caso pediátrico
}

\author{
Claudio Iglesias, ${ }^{1}$ Paul Puglia, ${ }^{2}$ Diego Pereira Núñez ${ }^{2}$ \\ ${ }^{1}$ Jefe de la unidad de Pediatría, Gastroenterología y Videoendoscopia. \\ ${ }^{2}$ Clínica de Cirugia Pediátrica, Facultad de Medicina UDELAR. \\ Centro Hospitalario Pereira Rossell, Montevideo, Uruguay.
}

Acta Gastroenterol Latinoam 2020;50(3):365-367

Recibido: 20/12/2018 / Aceptado: 18/09/2019 / Publicado online: 28/09/2020

\section{Resumen}

El hematoma duodenal intramural es una rara, pero conocida, complicación en la realización de una biopsia endoscópica. Es más común que se presente luego de un traumatismo abdominal, a maniobras terapéuticas endoscópicas, y es más frecuente en pacientes anticoagulados o con trastornos de la coagulación. La presentación clínica consiste en la aparición de los sintomas y los signos de una oclusión intestinal, una pancreatitis y una colestasis. El objetivo de esta presentación es comunicar el caso de una preescolar de cinco años, a quien se la realizó una biopsia duodenal endoscópica como parte del estudio para el diagnóstico de la enfermedad celíaca. Dicha paciente no presentaba antecedentes de sangrados ni trastornos de la coagulación. Como complicación desarrolló un hematoma duodenal intramural con una pancreatitis. Después reportamos la sintomatología presentada por la paciente, las maniobras diagnósticas y terapéuticas, su evo-

Correspondencia: Claudio Iglesias

Blvr. Artigas 1550 (CP: 11600), Piso 2, Pabellón Beisso, Centro Hospitalario Pereira Rossell. Montevideo, Uruguay

Tel.Fax: 0059827098091 / Cel.: 0059899883422

Correo electrónico: igleflo@gmail.com lución y advertimos a los endoscopistas pediatras que esta inusual complicación puede presentarse. Como conclusión, planteamos un caso de un hematoma duodenal intramural como una complicación de una biopsia endoscópica en una paciente sin antecedentes de sangrado ni trastornos de la coagulación. Dicho hematoma evolucionó a su resolución con un tratamiento conservador asistido por un equipo multidisciplinario constituido por un gastroenterólogo y un cirujano pediatra.

Palabras claves. Hematoma duodenal intramural, biopsia, endoscopia digestiva alta, pancreatitis.

\section{Intramural Duodenal hematoma after endoscopic biopsy: an unusual compli- cation. Paediatric case report}

\section{Summary}

The intramural duodenal hematoma is a rare but well-known collateral complication after an endoscopic biopsy. It is more frequent after abdominal trauma or therapeutic endoscopy and even more in anticoagulant-treated patients or those with coagulation disorders. Clinical presentation consists of the symptoms and the signs of the intestinal obstruction, the pancreatitis and the conjugated hyperbilirubinemia. We report a pediatric case of a five-year-old female patient who was being checked for celiac disease. Without known previous bleeding or coagulation disorders, she presented a intramural duodenal hematoma with pancreatitis as a complication of the endosco- 
pic biopsy procedure. A summary of the symptomatology and the signs, the diagnosis, the conservative treatment and evolution is described and also a reminder to paediatric endoscopists to be aware that this unusual complication can occur. This case presents the possibility that an intramural duodenal hematoma can be a complication of a duodenal endoscopic biopsy in patients with no coagulations disorders. This hematoma subsided with a non-surgical treatment (conservative), assisted by the joint work of a pediatric gastroenterologist and surgeon.

Key words. Intramural duodenal hematoma, biopsy, upper endoscopy, pancreatitis.

\section{Introducción}

El hematoma duodenal intramural (HDI) es una complicación colateral que tiene menor frecuencia en las biopsias endoscópicas que el trauma abdominal, el tratamiento endoscópico terapéutico como la inyección de epinefrina o polidocanol, y los métodos mecánicos (endoclips), que ya son entidades poco comunes. ${ }^{1-3}$ Los pacientes con tratamientos anticoagulantes o aquellos con trastornos de la coagulación tienen más riesgo de presentar un HDI. ${ }^{2,4}$ Es difícil establecer su frecuencia después de una biopsia endoscópica. Los centros que han reportado casos de un HDI informan que representan uno de cada 1120 a 3000 procedimientos completados., 5, 6 Ocurre más comúnmente en la población pediátrica y los adultos jóvenes. El $82 \%$ acontece en menores de 30 años. ${ }^{1,6}$

La presentación clínica incluye un dolor abdominal intenso y vómitos incesantes, asociados con la pancreatitis y la ictericia obstructiva. Las técnicas de imagenología como la ecografía y la tomografía computarizada son métodos utilizados en general para realizar el diagnóstico. ${ }^{3,7,8}$ La ecografía abdominal seriada es útil como un seguimiento y para confirmar la remisión del hematoma.?

El tratamiento es conservador, con el emplazamiento de una sonda nasogástrica con succión continua y la nutrición parenteral total, asistido por un gastroenterólogo endoscopista y un cirujano pediátrico, ya que la cirugía es una posibilidad terapéutica. ${ }^{9}, 10$

\section{Caso clínico}

Una niña de cinco años, caucásica, sin antecedentes clínicos personales o familiares a destacar, se sometió a una esofagogastroduodenoscopia (EGD) con el objetivo de obtener biopsias duodenales para confirmar la enfermedad celíaca. El procedimiento fue bien tolerado por la paciente que fue dada de alta ese mismo día en buenas condiciones.

Veinticuatro horas después del procedimiento, comenzó a tener vómitos y, después de una remisión de doce horas, se intensificaron en cantidad y frecuencia hasta que se volvieron incoercibles. Cuarenta y ocho horas después del procedimiento, empezó a sufrir un dolor abdominal cólico de intensidad moderada, sin fiebre y no se encontraron alteraciones urinarias o del tracto gastrointestinal inferior. La paciente fue nuevamente hospitalizada.

Del examen clínico se destacaron los síntomas y los signos de la debilidad, una deshidratación moderada, la palidez, la taquicardia y un llenado capilar lento. La paciente fue ingresada en la Unidad de Cuidados Intensivos.

Se le realizaron exámenes de laboratorio que dieron como resultado una alcalosis metabólica, un aumento de los glóbulos blancos (90\% polimorfonucleares), amilasa elevada (236 UI/L) y lipasa también alta (140 UI/L). La ecografía abdominal mostró una imagen ovoidea bien delimitada, hipoecoica de $50 \times 26$ × $26 \mathrm{~mm}$ de tamaño, con una ecotextura heterogénea y sin vascularización en la exploración Doppler, compatible con un hematoma de la segunda porción del duodeno (Figura 1). Esto se confirmó mediante una tomografía axial computarizada con y sin contraste intravenoso, estableciendo la ubicación no solo en la segunda, sino también en la tercera porción del duodeno y de un diámetro de $50 \mathrm{~mm}$ (Figura 2).

La paciente permaneció en la Unidad de Cuidados Intensivos y luego en la Unidad de Nutrición Enteral y Parenteral durante dieciséis días con una sonda nasogástrica y nutrición parenteral total. Durante el ingreso, hemoterapia descartó los trastornos de la coagulación. La niña fue controlada por un equipo multidisciplinario, compuesto por un gastroenterólogo pediátrico, un cirujano pediátrico y un ecografista en este período. Luego de recuperar la vía oral, fue dada de alta el día 19 en buenas condiciones.

Figura 1. La ecografía abdominal muestra un espacio sin ecos (flecha), correspondiente al hematoma duodenal intramural

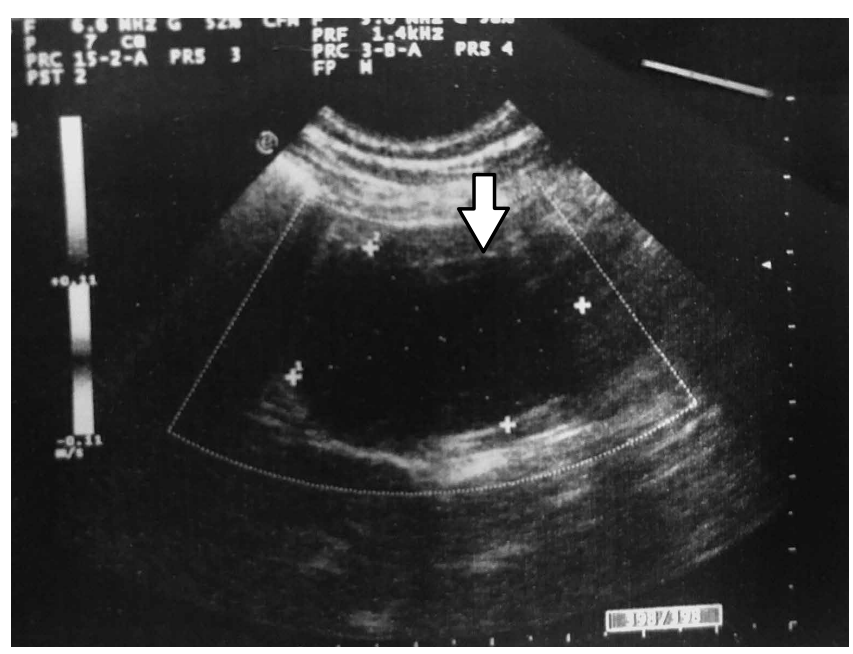


Figura 2. La tomografía computarizada abdominal muestra una masa en la segunda porción del duodeno (flecha), correspondiente al hematoma duodenal intramural

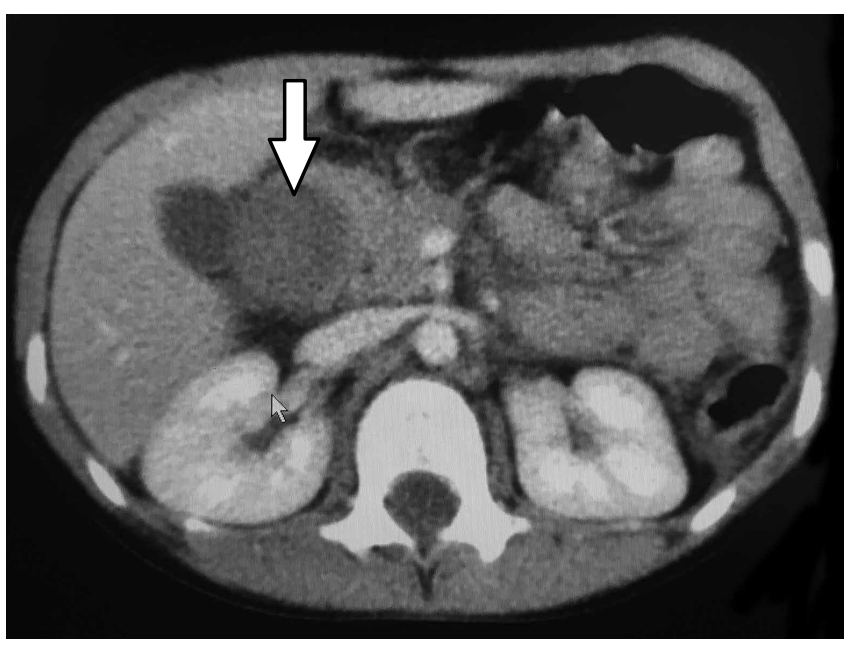

\section{Discusión}

Las complicaciones después de la EGD son raras y generalmente incluyen el sangrado y la perforación. Cuando el sangrado ocurre como una complicación luego de una biopsia duodenal, con mayor frecuencia, es intraluminal y es, por lo regular, autolimitado con complicaciones menores. Con menor frecuencia puede ser intramural, lo que resulta en un hematoma que al crecer ocluye la luz duodenal. También puede comprimir las estructuras adyacentes como los conductos biliares y los pancreáticos, lo que resulta en la ictericia y la pancreatitis. ${ }^{1}$ Algunas características anatómicas especiales del duodeno se han relacionado con esta complicación y también con el hecho de tomar las biopsias profundas no cercanas al endoscopio. Por lo tanto, Zinelis et al. sugirieron que al obtener las biopsias por pinzas no se extiendan a más de $2 \mathrm{o} 3 \mathrm{~cm}$ del endoscopio.?

Analizamos el caso de una niña de 5 años de edad, sin antecedentes personales ni familiares relevantes, sin trastornos de la coagulación, que presentó un HDI con una pancreatitis como complicación de una biopsia duodenal de rutina para la confirmación de la enfermedad celíaca. En el caso de nuestra paciente, los síntomas y signos ya descritos aparecieron dentro de las siguientes 48 horas después de la endoscopia. Estos son causados por la oclusión de la luz duodenal y la oclusión del tracto pancreático. La oclusión del tracto biliar también puede presentar hiperbilirrubinemia, lo que no se observó en este caso.

Es fundamental utilizar las técnicas de imagen como la ecografía y la tomografía computarizada para establecer el diagnóstico. ${ }^{3,8,9}$ En el caso presentado, la tomografía no solo confirmó la presencia del hematoma que se mues- tra en la ecografía, sino que también proporcionó nuevos datos sobre el tamaño y la ubicación.

Respecto al tratamiento, la mayoría de los autores sugieren un tratamiento conservador, colocando una sonda nasogástrica, la nutrición parenteral total y un tratamiento sintomático hasta la regresión total del hematoma y la pancreatitis. ${ }^{9} 10 \mathrm{Tal}$ método se realizó con éxito en nuestro caso, confirmando que el tratamiento conservador de estos pacientes es efectivo y el pronóstico del HDI con la pancreatitis es excelente.

\section{Conclusión}

Consideramos que el tratamiento interdisciplinario de estos pacientes es fundamental, ya que podrían requerir cirugía si no hay regresión del hematoma o si se presenta una perforación duodenal. Lo que no está definido es el tiempo de espera antes de la cirugía si no hay regresión del hematoma.

\section{Referencias}

1. Diniz-Santos DR, De Andrade Cairo RC, Braga H, Araújo-Neto C, Paes IB, Silva LR. Duodenal hematoma following endoscopic duodenal biopsy: A case report and review of the literature. Can J Gastroenterol 2006; 20 (1): 39-42.

2. Bechtel K, Moss RL, Leventhal JM, Spiro D, Abo A. Duodenal hematoma after upper endoscopy and biopsy in a 4-year-old girl. Pediatric Emergency Care 2006; 22 (9): 653-654.

3. Camarero C, Herrera D, Corbaton J, Mingo A, Olivares F, Roldan B. Intramural hematoma of the duodenum following endoscopic biopsy: an unusual complication of non-therapeutic endoscopy in children. Eur J Pediatr 2004; 163 (7): 418-419.

4. Chung S, Park CW, Chung HW, Shin SJ, Chang YS. Intramural duodenal hematoma and hemoperitoneum after endoscopic treatment in a patient with chronic renal failure on hemodialysis: a case report. Cases Journal 2009, 2: 9083.

5. Wang JY, Ma CJ, Tsai HL, Wu DC, Chen CY, Huang TJ, Hsieh JS. Intramural duodenal hematoma and hemoperitoneum in anticoagulant therapy following upper gastrointestinal endoscopy. Med Princ Pract 2006; 15 (6): 453-455.

6. Guzman C, Bousvaros A, Buonomo C, Nurko S. Intraduodenal hematoma complicating intestinal biopsy: case reports and review of the literature. The American Journal of Gastroenterology 1998; 93 (12): 2547-2550.

7. Zinelis SA, Hershenson LM, Ennis MF, Boller M, Ismail-Beigi F. Intramural duodenal hematoma following upper gastrointestinal endoscopy biopsy. Dig Dis Sci 1989; 34 (2): 289-291.

8. Hayashi K, Futagawa S, Kozaki S, Hirao K, Hombo Z. Ultrasound and CT diagnosis of intramural duodenal hematoma. Pediatr Radiol 1988; 18 (2): 167-168.

9. Dunkin D, Benkov KJ, Rosenberg HK. Duodenal and rectal hematomas complicating endoscopic biopsy: use of sonography in pediatrics. J Ultrasound Med 2009; 28 (11): 1575-1580.

10. Winckworth LC, Tricot T, Fertleman C, Blumberg R. An unusual complication of endoscopic intestinal biopsy. Journal of Paediatrics and Child Health 2011; 47 (11): 842. 